\title{
Pesquisa científica no curso de Enfermagem: revisão integrativa
}

\section{Scientific research on the Nursing graduation: an integrative review}

\author{
${ }^{1}$ Marcela Pereira Oliveira marcelaoliveira727@gmail.com \\ 2 Ilda Cecília Moreira da Silva \\ ${ }^{2}$ Gabriela Girão Albuquerque
}

\section{RESUMO}

O curso de enfermagem vem buscando modificar o perfil do profissional, através de uma formação técnica, científica, crítica e reflexiva por intermédio da pesquisa científica. Porém, parte desses alunos são desestimulados ao realizar essa atividade. Este trabalho teve como objetivo buscar evidências cientificas sobre a percepção do aluno de enfermagem acerca da pesquisa científica. Utilizou-se como metodologia a revisão integrativa. A pesquisa foi realizada em bases de dados na área da saúde e os descritores utilizados foram:estudantes de enfermagem e pesquisa em enfermagem. Foram encontrados 15 artigos.Percebeu-se que os alunos entendem a importância da pesquisa científica na formação e no desenvolvimento acadêmico e profissional. A falta de integração entre instituições de ensino superior e os serviços de saúde foi apontada como dificuldade para realização e elaboração de pesquisas cientificas. As instituições de ensino superior, as atividades de extensão e o apoio docente foram apontados como essenciais no estímulo do aluno ao ato constante da pesquisa. Considera-se a necessidade da mudança do perfil do professor para melhor desenvolvimento do aluno e a busca por metodologias e instrumentos para o estímulo do estudo e do desenvolvimento da prática em questão. Além disso, melhor integração ensino com os serviços de saúde.

Palavras-chave: Estudantes de Enfermagem, Pesquisa Científica, Metodologia.

\begin{abstract}
The Nursing school is changing the professional profile, through technical training, scientific, critical and reflective through scientific research. But some of these students are discouraged to carry out these activites. This study aimed to search scientific evidence on the perception of nursing students about scientific research. It was used as methodology the integrative review. The survey was conducted in databases health and descriptors used were: nursing students and nursing research. 15 articles were found. It was noticed that students understand the importance of scientific research in training and academic and professional development. The lack of integration of education institutions with health services was identified hinder in the achievement of research. The support higher education institutions, extension activities and stimulus of the professor were identified as essential in encouraging the student to the constant research act. It is considered changing the professor's and profile for better development of the student and the search for methods and instruments for stimulating the study and the development of the practice in question. More over better integration education with ]health service.
\end{abstract}

Keywords: NursingStudents, ScientificResearch, Methodology.

1 Enfermeira. Especialista em Enfermagem do Trabalho. Mestranda em Ensino em Ciências da Saúde e Meio Ambiente pelo Centro Universitário de Volta Redonda.

2 Centro Universitário de Volta Redonda, UniFOA. 


\section{INTRODUÇÃO}

As crescentes demandas e complexidades da vida social exigem, cada vez mais, a formação de profissionais competentes, que buscam atualizar-se continuamente e a incorporação da atividade da pesquisa ao cotidiano profissional voltado para a construção e a ampliação dos conhecimentos (NAJJAR, 2009).

As pesquisas científicas na área da enfermagem estão crescendo em todo o mundo, principalmente em nível nacional. Porém, quando comparadas à outras áreas nota-se a necessidade de avançar em pesquisas mais qualificadas (PIEXAK et al., 2013).

Normalmente ao final da graduação o acadêmico tem como dever a construção de um Trabalho de Conclusão de Curso (TCC),no qual é orientado por um professor. Desta forma, na maior parte dos casos, o acadêmico se depara com um ambiente de pesquisa, no qual nunca tinha experimentado antes. O TCC tem como finalidade introduzir o aluno à pesquisa, fazendo-o utilizar a metodologia científica para detectar, conhecer, resolver situações e propor ações que necessitam da intervenção da enfermagem, além de incentivá-lo a prosseguir com sua formação acadêmica (HEYDEN et al., 2003). Porém, o TCC raramente é visto pelos acadêmicos como uma etapa proveitosa, no qual se dá por uma série de fatores como: escassez de tempo, dificuldade na construção do referencial teórico; dificuldade com o desenho da pesquisa (FREITAS et al., 2013).

A problemática levantada neste estudo é que os acadêmicos de enfermagem apresentam dificuldades ao realizar pesquisas científicas e estas, somente são observadas pelos docentes quando o aluno realiza o TCC. Diante dessas considerações, é perceptível que a concepção do aluno a respeito desta temática é fundamental para que o corpo docente aprimore ou elabore novas estratégias de ensino-aprendizagem que induza o aluno ao ato contínuo da pesquisa em sua vivência acadêmica e posteriormente profissional.

No entendimento de Martins (2008), a investigação apoia o desenvolvimento contínuo da enfermagem, a tomada de decisões inteligentes e adequadas para prestar assistência de qualidade, vincular os conhecimentos empíricos e científicos da profissão, solidificando o nível do saber e contribuindo para sua visibilidade social. A pesquisa na graduação favorece o futuro do enfermeiro, o conhecimento crítico, a capacidade de resolver os problemas da prática profissional através da pesquisa (GOMES; SANNA, 2004).

Este estudo teve como objetivo identificar a percepção do estudante de enfermagem acerca da pesquisa científica através da análise deartigos científicos disponíveis em bases de dados. Tal investigação se justifica pela importância de preparar os acadêmicos e futuros profissionais para os problemas e barreiras que ocorrem no dia a dia, buscando soluções cabíveis na pesquisa.

\section{METODOLOGIA}

Para atingir os objetivos deste estudo foi utilizado o método de Revisão Integrativa da Literatura. Este método se concentra em uma ampla e rigorosa síntese de estudos que abordam o problema a ser investigado (SOUZA; SILVA; CARVALHO, 2010). Para realizar este tipo de estudo faz-se necessário seguir etapas. De acordo com Mendes; Silveira; Galvão (2008) são essas:

- primeira etapa: identificação do tema, seleção da questão para revisão;

- segunda etapa: estabelecimento de critérios para inclusão e exclusão de estudos/ amostragem ou busca na literatura;

- terceira etapa: definição das informações a serem extraídas dos estudos selecionado; 
- quarta etapa: categorização dos dados obtidos;

- quinta etapa: avaliação dos estudos selecionados;

- sexta etapa: apresentação da revisão/síntese do conhecimento.

Levando em consideração os desestímulosapresentados pelo acadêmico de enfermagem ao realizar pesquisas científicas, surgiram as seguintes questões norteadoras:

- Qual a percepção do estudante de enfermagem acerca da pesquisa científica?

- Quais dificuldades são encontradas pelos estudantes de enfermagem ao realizar pesquisa científica?

- Como estimular o aluno de enfermagem a iniciar precocemente a realização de pesquisas científicas?

Para a busca das evidências foram utilizados os seguintes critérios de inclusão: artigos científicos disponíveis nas bases de dados Base de Dados de Enfermagem (BDENF), Literatura Latino-Americana e do Caribe (LILACS) e Biblioteca Científica Eletrônica Online(Scielo) que abordassem o tema pesquisa científica no curso de enfermagem, publicadas em inglês, português ou espanhol. Foram utilizadosmétodos de busca avançada de acordo com as especificidades de cada base de dados cruzando seguintes descritores: "Estudantes de enfermagem" e "Pesquisa em enfermagem".

Foram excluídos artigos que não se encontravam na íntegra e além disso, apesquisa foi realizada de forma ordenada, respectivamente: BDENF, SCIELO e LILACS e desta maneira as publicações que se encontravam indexadas em mais de uma, foram selecionadas na primeira busca.

Os resultados dos artigos foram apresentados em forma descritiva, fazendo uso de quadro, objetivando expor as evidências acerca do ato da pesquisa científica no curso de enfermagem.

Após a utilização dos critérios de inclusão e exclusão foram pré-selecionados 29 artigos na BDENF, 1 artigo no SCIELO e 16 artigos no LILACS, totalizando 46 artigos científicos.

\section{RESULTADOS E DISCUSSÃO}

Após a leitura dos artigos, foram incluídos neste trabalho 15 artigos dos 46 pré-selecionados. Dos 15 artigos publicados sobre a temática pode-se observar que durante os anos 2014 e 2015, não foram publicadas evidências até o momento da revisão. Ademais, os artigos selecionados para composição do estudo foram distribuídos dentre os anos de 2005 a 2013 e foram eleitos 2 artigos de 1998, devido sua relevância sobre o tema. O ano que se destacou com maior número de artigos publicados foi o ano de 2009.

Este aumento do número de artigos em 2009, pode estar relacionado as inúmeras mudanças que ocorreram nas Diretrizes Curriculares Nacionais no curso de Enfermagem. De acordo com o Conselho Nacional de Educação sob a resolução CNE/CES n ${ }^{\circ} 3$ de 7de novembrode 2001 em seu artigo $3^{\circ}$ diz que: o "curso de enfermagem tem como perfil do formado egresso/profissional: enfermeiro com formação generalista, humanista, crítica e reflexiva. Profissional qualificado para o exercício de Enfermagem pautado no rigor técnico científico”.

Em relação aos periódicos, destacamos o maior número de artigos publicados na Revista Brasileira de Enfermagem (REBEN) e na Escola Anna Nery Revista de Enfermagem.Vale ressaltar que a Revista Brasileira 
de enfermagem é o mais antigo periódico de enfermagem no Brasil, órgão público da Associação Brasileira de Enfermagem (ABEN). E a Escola Anna Nery uma das mais importantes escolas de enfermagem do Brasil.

No Quadro 1 apresentado abaixo, pode-se verificar os artigos selecionados para este estudo, apresentando autores, título, ano da publicação e contribuição da pesquisa.

\section{Quadro 1 - Distribuição dos estudos segundo Autor (es), Título, Periódico, Ano de Publicação e Contribuição da Pesquisa}

\begin{tabular}{|c|c|c|c|c|}
\hline Autor (res) & Título & Periódico & $\begin{array}{l}\text { Ano de } \\
\text { Publicação }\end{array}$ & Contribuição da pesquisa \\
\hline Piexak et al. & $\begin{array}{l}\text { A percepção de estudantes de } \\
\text { enfermagem da primeira série de um } \\
\text { curso de graduação em enfermagem } \\
\text { acerca da pesquisa }\end{array}$ & $\begin{array}{l}\text { Esc Anna } \\
\text { Nery Rev } \\
\text { Enf }\end{array}$ & 2013 & $\begin{array}{l}\text { Os alunos percebem a pesquisa em } \\
\text { Enfermagem como imprescindível } \\
\text { para formação profissional }\end{array}$ \\
\hline $\begin{array}{l}\text { Santos; Anjos; } \\
\text { Almeida }\end{array}$ & $\begin{array}{l}\text { A percepção de formandos sobre a } \\
\text { pesquisa em enfermagem no curso } \\
\text { de graduação }\end{array}$ & $\begin{array}{l}\text { RevEnferm } \\
\text { UFSM }\end{array}$ & 2013 & $\begin{array}{l}\text { A Instituição de Ensino Superior } \\
\text { utliza pouco da prática da pesquisa } \\
\text { para contruibuir para formação } \\
\text { pessoal e na melhoria das condições } \\
\text { de saúde. }\end{array}$ \\
\hline Fortuna et al. & $\begin{array}{l}\text { A pesquisa e articulação ensino- } \\
\text { serviço na consolidação do Sistema } \\
\text { Único de Saúde }\end{array}$ & $\begin{array}{l}\text { RevEsc } \\
\text { Enferm USP }\end{array}$ & 2011 & $\begin{array}{l}\text { A articulação do ensino e serviço } \\
\text { é desafiadora e complexa. Há } \\
\text { necessidade de recusa em que separa } \\
\text { quem pesquisa, o que ou quem é } \\
\text { pesquisado, quem aprende, ensina e } \\
\text { cuida. }\end{array}$ \\
\hline $\begin{array}{l}\text { Silva; Daves; } \\
\text { Cunha }\end{array}$ & $\begin{array}{l}\text { Pesquisa Científica em Enfermagem } \\
\text { sob ótica docente e discente }\end{array}$ & $\begin{array}{l}\text { Rev de Pesq: } \\
\text { Cuidado é } \\
\text { fundamental }\end{array}$ & 2010 & $\begin{array}{l}\text { A importância da articulação } \\
\text { do ensino, pesquisa e extensão. } \\
\text { A pesquisa é indissociavel para } \\
\text { formação de um indivíduo crítico. }\end{array}$ \\
\hline López et al. & $\begin{array}{l}\text { Aprendizagem baseada em } \\
\text { problemas em Enfermagem } \\
\text { Comunitária I }\end{array}$ & Enf UERJ & 2010 & $\begin{array}{l}\text { Detectou qualificação notável em } \\
\text { mais da metade do alunado. }\end{array}$ \\
\hline Erdman et al. & $\begin{array}{l}\text { Vislumbrando o significado da } \\
\text { iniciação científica a partir do } \\
\text { graduando de enfermagem }\end{array}$ & $\begin{array}{l}\text { Esc Anna } \\
\text { Nery Rev } \\
\text { Enf }\end{array}$ & 2010 & $\begin{array}{l}\text { Desvelou a iniciação científica } \\
\text { como um processo de crescimento, } \\
\text { mudança, e aprendizagem na } \\
\text { graduação da enfermagem e } \\
\text { continuidade na academia. }\end{array}$ \\
\hline Krahl et al. & $\begin{array}{l}\text { Experiência dos acadêmicos de } \\
\text { enfermagem em um grupo de } \\
\text { pesquisa }\end{array}$ & RevBras Enf & 2009 & $\begin{array}{l}\text { Promoveu enriquecimento no } \\
\text { processo de ensino-aprendizagem e } \\
\text { a partir de um forma parcipativa, } \\
\text { apropiaram-se, produziram e } \\
\text { difundiram o conhecimento, indo } \\
\text { além do processo cognitivo. }\end{array}$ \\
\hline Backes et al. & $\begin{array}{l}\text { Grupos de Pesquisa de Educação em } \\
\text { Enfermagem da região sul do Brasil }\end{array}$ & $\begin{array}{l}\text { Rev Gaúcha } \\
\text { de Enf }\end{array}$ & 2009 & $\begin{array}{l}\text { Os principais desafios são: falta de } \\
\text { interdisciplinariedade, integração } \\
\text { ensino-serviço e de fomento de bolsas } \\
\text { para o avanço da área da educação }\end{array}$ \\
\hline Miguéis et al. & $\begin{array}{l}\text { Da prática de pesquisa à prática } \\
\text { de formação para o cuidado de } \\
\text { enfermagem: um estudo de caso }\end{array}$ & $\begin{array}{l}\text { Online } \\
\text { Brazilian } \\
\text { Journal of } \\
\text { Nursing } \\
\end{array}$ & 2009 & $\begin{array}{l}\text { Através da pesquisa os alunos } \\
\text { internalizaram os conceitos de } \\
\text { cuidado. }\end{array}$ \\
\hline Kirchhof et al. & $\begin{array}{l}\text { Coleta de dados: uma experiência } \\
\text { para o ensino e a pesquisa }\end{array}$ & $\begin{array}{l}\text { Cogitare } \\
\text { Enferm }\end{array}$ & 2009 & $\begin{array}{l}\text { As experiência teório-práticas } \\
\text { oportunizadas pelo currículo da } \\
\text { graduação alcançaram um diferencial } \\
\text { no processo do conhecimento. }\end{array}$ \\
\hline
\end{tabular}




\begin{tabular}{|l|l|l|l|l|}
\hline Autor (res) & Título & Periódico & $\begin{array}{l}\text { Ano de } \\
\text { Publicação }\end{array}$ & Contribuição da pesquisa \\
\hline $\begin{array}{l}\text { Palmeira e } \\
\text { Rodriguéz }\end{array}$ & $\begin{array}{l}\text { A investigação científica no curso de } \\
\text { enfermagem: uma análise crítica }\end{array}$ & $\begin{array}{l}\text { Esc Anna } \\
\text { Nery Rev } \\
\text { Enf }\end{array}$ & 2008 & $\begin{array}{l}\text { É necessário estimular e explorar } \\
\text { o gosto pela investigação nos } \\
\text { professores e nos alunos, para que } \\
\text { essa atividade converta-os em eternos } \\
\text { aprendizes da prática investigativa. }\end{array}$ \\
\hline Ferreira & $\begin{array}{l}\text { Metodologia de Pesquisa : uma } \\
\text { experiência com alunos do curso de } \\
\text { graduação em enfermagem }\end{array}$ & $\begin{array}{l}\text { Rev Baiana } \\
\text { em Enf }\end{array}$ & 2007 & $\begin{array}{l}\text { É muito mais fácil estudar, aprender e } \\
\text { pesquisar quando se é estimulado. }\end{array}$ \\
\hline $\begin{array}{l}\text { Camponogara } \\
\text { et al. }\end{array}$ & $\begin{array}{l}\text { Espaço de Diálogo na Pesquisa } \\
\text { em Enfermagem: um relato de } \\
\text { experiência sobre a fase de coleta } \\
\text { de dados }\end{array}$ & $\begin{array}{l}\text { Texto e } \\
\text { Contexto }\end{array}$ & $\begin{array}{l}\text { O ato de pesquisar em enfermagem } \\
\text { deve envolver, principalmente, } \\
\text { técnica, afetividade, sensibilidade, } \\
\text { coragem e ética, resultando em } \\
\text { enfermeiros motivados e o avanço da } \\
\text { profissão. }\end{array}$ \\
\hline $\begin{array}{l}\text { Rodrigues e } \\
\text { Cassiani }\end{array}$ & $\begin{array}{l}\text { Atitudes acerca da pesquisa } \\
\text { científica entre estudantes de } \\
\text { enfermagem de três escolas do } \\
\text { interior do estado de São Paulo }\end{array}$ & $\begin{array}{l}\text { Rev Bras } \\
\text { Enf }\end{array}$ & $\begin{array}{l}\text { A Realidade da Pesquisa no DEN/ } \\
\text { UFS }\end{array}$ & $\begin{array}{l}\text { Os alunos possuem atitudes } \\
\text { responsáveis frente à pesquisa e } \\
\text { concordam que é fundamental ao } \\
\text { aluno a prática de investigação } \\
\text { científica junto de seus professores. }\end{array}$ \\
\hline Alves e Santos & $\begin{array}{l}\text { Necessidade de construção grupos } \\
\text { de pesquisa pelos docentes e maior } \\
\text { disponibilidade dos mesmos para esta } \\
\text { atividade. }\end{array}$ \\
\hline
\end{tabular}

Fonte: dos autores.

Dentre os artigos analisados todos os estudos revelaram a vantagem da pesquisa no aperfeiçoamento do aluno e no desenvolvimento profissional. Concomitantemente, os artigos mostraram que o aluno entende a importância da pesquisa para o seu progresso e para visibilidade da enfermagem.

A produção acadêmica então se faz não somente necessária, mas capaz de tornar o aluno crítico e protagonista do seu próprio conhecimento, buscando a resolução dos problemas acadêmicos e/ou profissionais nas evidências.

Amaral (2010), colabora quando em seu estudo buscou o entendimento da pesquisa pelo aluno e afirma que os discentes após o contato com a pesquisa tiveram uma percepção diferente, passando a compreender a necessidade da prática para o enriquecimento e contribuição profissional.

Além disso, essa prática torna possível encontrar o conhecimento de punhos próprios e permite os discentes a conhecer conteúdos importantes e inovadores e auxilia na formulação de críticas a respeito dos mesmos (SIQUEIRA, 2010).

Observou-se na revisão integrativa que é um grande desafio os serviços de saúde estarem articulados com o ensino e assim, fomentando a pesquisa. A pesquisa científica está inserida na área acadêmica e afastada dos serviços de saúde e o maior desafio dos grupos de pesquisa de enfermagem diz respeito à coparticipação das instituições de saúde no campo da prática assistencial (TRENTINI; SILVA, 2012)

A produção científica está inserida na área acadêmica e distante dos serviços de saúde e assim exercendo pouco valimento na construção de políticas de saúde, é primordial um esforço coletivo para elaboração de projetos e pesquisas que simultaneamente produzam conhecimentos e transformem as práticas de saúde. (FORTUNA; MISHIMA, 2012). 
Em outros estudos desta revisão, as atividades extensionistas, o apoio do docente e das instituições de ensino superior foramreferidoscomo condições relevantes para impulsionar a prática da pesquisa.

A Extensão Universitária é compreendida como atividade que contribui para o processo de formação acadêmica, através da vivência dos alunos a partir de ações sociais que promovem trocas e associações com a realidade ali praticada e estudada. Nessas atividades os alunos são entendidos como agentes de transformações sociais (FORPROEX, 2006).

Biscarde, Santos e Silva (2014), afirmam que as ações extensionistas são fundamentais na formação acadêmica e contribuem para o desenvolvimento do aprendizado do aluno, muito além daqueles apreendidos nos moldes tradicionais e bancários de formação de conhecimento.

De acordo com a Lei de Diretrizes e Bases - LDB (1996), artigo 43, o ensino superior deve estimular a prática da pesquisa e investigação científica buscando desenvolver a ciência, a tecnologia, criação e difusão da cultura.

Apesar da importância das novas metodologias de ensino aprendizagem, ainda encontramos professores que utilizam somente slides para apresentação e desenvolvimento de conteúdo, fazendo com o que aprendizado fique engessado nas velhas formas de aprender, não inovando e não pesquisando.

No ano de 2002 em um fórum de reflexão universitária na UNICAMP, foi afirmado que a qualidade da pesquisa depende da essência de seus pesquisadores. Ainda, relatam que é imprescindível contratar bons profissionais, levando em conta os objetivos institucionais.

\section{CONSIDERAÇÕES}

O curso de enfermagem por anos foi visto como um processo de cuidar, sem o olhar científico e por muitas vezes se calou frente as situações que são competentes a ela, não mostrando a riqueza e a importância deste profissional na área da saúde.

É oportuno frisar que há necessidade de maior integração entre os serviços de saúde e as instituições de ensino. É preciso propor a união para que aconteça o desenvolvimento acadêmico através da prática baseada em evidências e assim, o aperfeiçoamento e a melhora da assistência prestada para com o ser humano.

A partir deste estudo, encontramos a importância do elo do ensino, pesquisa e extensão para que o aluno reconheça e valorize a pesquisa. As atividades de extensão se tornaram um instrumento adequado para a inserção do aluno na realidade social e além disso, aguça a execução de pesquisas direcionadas a solução de problemas encontradas na realização dessas atividades.

Outro fator relevante, é o papel do docente enquanto incentivador desta prática. Não é eficaz investir em práticas quando não se tem o docente trabalhando dentro dos princípios da instituição de ensino superior. Necessita-se de profissionais com perfil arrojado, com métodos inovadores e que busquem na essência dos alunos a melhor metodologia para o desenvolvimento do aprendizado.

Faz-se necessário a aproximação real do docente e do aluno quando se trata de produção científica. O docente deve se perceber como multiplicador de ideias e práticas, não apenas transmissor de conteúdo. 


\section{REFERÊNCIAS}

AMARAL, R. As contribuições da pesquisa científica na formação acadêmica. Identidade Científica, Presidente Prudente-SP, v. 1, n. 1, p. 64-74, jan./jun. 2010.

BISCARDE, D.G. S; PEREIRA-SANTOS, M; SILVA, L.B. Formação em saúde, extensão universitária e Sistema Único de Saúde (SUS): conexões necessárias entre conhecimento e intervenção centradas na realidade e repercussões no processo formativo. Interface (Botucatu),Botucatu , v. 18, n. 48, p. 177-186, 2014.

BRASIL. Brasília. Resolução CNE/CES n ${ }^{\circ} 3$ de Novembro de 2001. Diretrizes Curriculares Nacionais do Curso de Graduação de em Enfermagem. Diário Oficial da União, Brasília, 9 de Novembro de 2001. Seção 1, p. 37.

BRASIL. Lei Darcy Ribeiro (1996). LDB: Lei de diretrizes e bases da educação nacional [recurso eletrônico]: Lei $n^{\circ}$ 9.394, de 20 de dezembro de 1996, que estabelece as diretrizes e bases da educação nacional. - 9 . ed. - Brasília: Câmara dos Deputados, Edições Câmara, 2014. 45 p. - (Série legislação; n. 118).

GOMES, M.M.F; SANNA, M.C. A pesquisa em enfermagem no congresso de iniciação científica de uma universidade de São Paulo. Rev. bras. Enferm.Brasília , v. 57, n. 5, p. 574-578, out. 2004.

HEYDEN, M.S.T; RESCK, Z.M.R; GRADIM, C.V.C. A pesquisa na graduação em enfermagem: requisito para conclusão do curso. Rev. bras. enferm. Brasília , v. 56, n. 4, Aug. 2003.

FORPROEX - Fórum De Pró-Reitores De Extensão Das Universidades Públicas Brasileiras.Indissociabilidade ensino-pesquisa-extensão e a flexibilização curricular: uma visão da extensão. Brasília: MEC/SESu, 2006.

FORTUNA, C.M; MISHIMA S.M . A pesquisa de enfermagem e a qualificação da assistência: algumas reflexões. Rev. Eletr. Enf. 2012 14(4):740-2 .

FÓRUM DE REFLEXÃO UNIVERSITÁRIA. Os desafios da pesquisa no Brasil. Caderno Temático. Suplemento Jornal da Unicamp. Campinas, ano I, n 12, Fev 2002.

FREITAS SMB et al. Dificuldades vivenciadas na construção do tcc: percepção de estudantes egressos de um curso de graduação em enfermagem. IN: SEMINARIO NACIONAL DE PESQUISA EM ENFERMAGEM,2013. Anais do 17 Seminario Nacional de Pesquisa em enfermagem, Natal, 2013.

MARTINS,J.C.M. Investigações em Enfermagem: alguns apontamentos sobre dimensão ética. Pensar Enfermagem. Lisboa, v.12, n.2, 2008.

MENDES,K.D.S ; SILVEIRA, R.C.C.P ; GALVÃO, C.M . Revisão Integrativa: método de pesquisa para incorporação de evidências na saúde. Texto contexto - enferm. vol.17 no.4 Florianópolis Oct./Dec. 20088.

NAJJAR, E.C.A; ALVES, M.A.S. Competências e habilidades para pesquisa em alunos degraduação de terapia ocupacional. Ciências e Cognição 2009; Vol 14 (3): 145-159.

PIEXAK, D.R et al . A percepção de estudantes da primeira série de um curso de graduação em enfermagem acerca da pesquisa. Esc. Anna Nery, Rio de Janeiro , v. 17, n. 1, Mar. 2013.

SIQUEIRA, M. A Relevância da Pesquisa Científica na formação dos discentes. FIDES, Natal, v. 1, n. 1, fev.jul. 2010.

SOUZA, M.T; SILVA M.D; CARVALHO, R. Revisão Integrativa: o que é e como fazer. Einstein, São Paulo, v.8, n.1. 2010, p 102-6.

TRENTINI, M; SILVA, D.G.V. Grupos de pesquisa em enfermagem: a transferibilidade do conhecimento para a prática. Texto contexto - enferm. Florianópolis, v. 21, n. 4, Dez. 2012. 